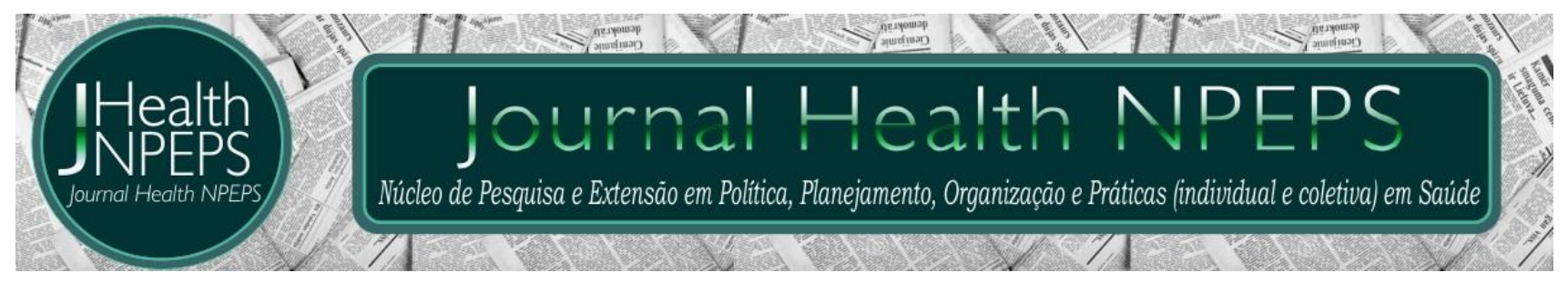

http://dx.doi.org/10.30681/252610104960

ARTIGO ORIGINAL

\title{
Atitude dos enfermeiros gestores face à morte: repercussões da pandemia por COVID-19
}

\section{Nursing managers' attitudes towards death: repercussions of the COVID-19 pandemic}

\author{
Actitudes de los gerentes de enfermería ante la muerte: repercusiones de la \\ pandemia COVID-19
}

\begin{abstract}
Maria Filomena Passos Teixeira Cardoso ${ }^{1}$, Maria Manuela Ferreira Pereira da Silva Martins², Olga Maria Pimenta Lopes Ribeiro3 ${ }^{3}$, Virgínia Lucinda Sousa Cruz Pereira ${ }^{4}$, Regina Maria Ferreira Pires ${ }^{5}$, Margarida Reis Santos ${ }^{6}$
\end{abstract}

\section{RESUMO}

Objetivo: analisar as atitudes dos enfermeiros gestores face à morte, antes e após o período crítico da pandemia por COVID-19. Método: estudo quantitativo, transversal e comparativo, realizado em uma instituição hospitalar do Norte de Portugal. A coleta de dados ocorreu através de questionário, que integrava a Escala de Avaliação do Perfil de Atitudes acerca da Morte, em dois momentos. O primeiro ocorreu em 2018, com participação de 28 enfermeiros gestores, e o segundo, em 2020, com 21 enfermeiros gestores. Resultados: globalmente, os participantes manifestaram maior concordância com as atitudes "aceitação neutral/neutralidade" e "medo". Embora algumas das cinco

\footnotetext{
${ }^{1}$ Enfermeira. Doutoranda em Ciências de Enfermagem. Enfermeira Diretora do Centro Hospitalar Universitário São João. Docente na Universidade Fernando Pessoa, Departamento de Enfermagem. Porto, Portugal. E-mail: ptcardoso@gmail.com ORCID ID: http://orcid.org/0000-0001-5758-2310

${ }^{2}$ Enfermeira. Doutora em Ciências de Enfermagem. Professora Coordenadora na Escola Superior de Enfermagem do Porto. Investigadora integrada no Centro de Investigação em Tecnologias e Serviços de Saúde (CINTESIS). Porto, Portugal. E-mail: mmartins@esenf.pt ORCID ID: http://orcid.org/0000-0003-1527-9940

${ }^{3}$ Enfermeira. Doutora em Ciências de Enfermagem. Professora Adjunta na Escola Superior de Enfermagem do Porto. Investigadora integrada no Centro de Investigação em Tecnologias e Serviços de Saúde (CINTESIS). Porto, Portugal. Email: olgaribeiro@esenf.pt ORCID ID: http://orcid.org/0000-0001-9982-9537 Autor para correspondência Endereço: Rua Dr. António Bernardino de Almeida, 830, 844, 856, 4200-072, Porto, Portugal.

${ }^{4}$ Enfermeira. Mestre em Enfermagem de Reabilitação. Enfermeira em funções de gestão no Centro Hospitalar Universitário São João. Porto, Portugal. E-mail: vpereiraenf@gmail.com ORCID ID: http://orcid.org/0000-0002-4884$\underline{3627}$

${ }^{5}$ Enfermeira. Doutora em Ciências de Enfermagem. Professora Adjunta na Escola Superior de Enfermagem do Porto. Porto, Portugal. E-mail: regina@esenf.pt ORCID ID: http://orcid.org/0000-0003-1610-7091

${ }^{6}$ Enfermeira. Doutora em Ciências de Enfermagem. Professora Coordenadora na Escola Superior de Enfermagem do Porto, Porto, Portugal. Investigadora integrada no Centro de Investigação em Tecnologias e Serviços de Saúde (CINTESIS). Porto, Portugal. E-mail: mrs@esenf.pt ORCID ID: http://orcid.org/0000-0002-7948-9317
}

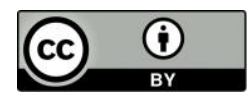

Este artigo está licenciado sob forma de uma licença Creative Commons Atribuição 4.0 Internacional, que permite uso irrestrito, distribuição e reprodução em qualquer meio, desde que a publicação original seja corretamente citada. 
dimensões das atitudes não tenham apresentado diferenças significativas entre o primeiro e segundo momento, constatou-se que em relação à "aceitação como aproximação", a média obtida no momento, após o período crítico da pandemia por COVID-19, foi superior. Conclusão: a partir dos resultados emerge a importância de se investir na preparação dos enfermeiros gestores para lidar com a morte e o processo de morrer, com uma dupla intencionalidade: minimizar o seu sofrimento e assegurar a otimização do acompanhamento e apoio aos enfermeiros da equipe que lidera.

Descritores: Atitude Frente a Morte; Enfermagem; Infeções por Coronavírus; Pandemias; Gestor de Saúde.

\section{ABSTRACT}

Objective: to analyze the attitudes of nurse managers towards death, before and after the critical period of the pandemic by COVID-19. Method: quantitative, cross-sectional and comparative study, carried out in a hospital in Northern Portugal. Data collection occurred through a questionnaire, that integrated the Attitude Profile Assessment Scale about Death, in two stages. The first occurred in 2018, with the participation of 28 nurse managers, and the second, in 2020, with 21 nurse managers. Results: globally, the participants showed greater agreement with the attitudes of "neutral acceptance/neutrality" and "fear". Although some of the five dimensions of attitudes did not show significant differences between the first and second moments, it was found that in relation to "acceptance as approximation", the average obtained at the moment, after the critical period of the pandemic by COVID-19, was superior. Conclusion: from the results emerges the importance of investing in the preparation of nurse managers to deal with death and the process of dying, with a double intention: to minimize their suffering and ensure the optimization of monitoring and support to the nurses of the team that leads.

Descriptors: Attitude to Death; Nursing; Coronavirus Infections; Pandemics; Health Manager.

\section{RESUMEN}

Objetivo: analizar las actitudes de los gerentes de enfermería hacia la muerte, antes y después del período crítico de la pandemia por COVID-19. Método: estudio cuantitativo, transversal y comparativo, realizado en un hospital del norte de Portugal. La recolección de datos se realizó a través de un cuestionario, que integró el Escala de Evaluación del Perfil de Actitud sobre la Muerte, en dos etapas. El primero ocurrió en 2018, con la participación de 28 gerentes de enfermería, y el segundo, en 2020, con 21 gerentes de enfermería. Resultados: globalmente, los participantes mostraron mayor acuerdo con las actitudes de "aceptación neutral/neutralidad" y "miedo". Si bien algunas de las cinco dimensiones de las actitudes no mostraron diferencias significativas entre el primer y segundo momento, se encontró que en relación a la "aceptación como aproximación", el promedio obtenido en el momento, luego del período crítico de la pandemia por COVID-19, fue superior. Conclusión: de los resultados surge la importancia de invertir en la preparación de los gerentes de enfermería para afrontar la muerte y el proceso de morir, con una doble intención: minimizar su sufrimiento y asegurar la optimización del seguimiento y acompañamiento a los enfermeros del equipo que lideran.

Descriptores: Actitud Frente a la Muerte; Enfermería; Infecciones por Coronavirus; Pandemias; Gestor de Salud. 


\section{INTRODUÇÃO}

Atualmente, perante as exigências relacionadas com a qualidade em saúde, as instituiçõos hospitalares se deparam com a necessidade de dar respostas efetivas a desafios de alta complexidade e diferenciação ${ }^{1}$. A qualidade da assistência prestada pelos profissionais assume maior relevância, em contexto pandêmico, quando as pessoas que recorrem aos serviços de saúde se encontram em uma condição de fragilidade $^{2}$, comprometedora de uma tomada de decisão efetiva.

A atual pandemia pela COVID-19 se apresentou como um desafio para todos os gestores, nomeadamente os enfermeiros gestores, que perante esta situação inesperada, tiveram de adotar estratégias que garantissem a segurança dos clientes e profissionais, bem como o desenvolvimento das melhores práticas $^{3,4}$. Além da adequação das condições estruturais, os enfermeiros gestores têm um papel determinante na gestão dos seus colaboradores e de si próprios, de forma a conseguirem manter a sua atividade e garantir 0 máximo de segurança e bem-estar, quer para os profissionais, quer para os clientes $^{4}$. Importa ainda destacar, no âmbito do exercício profissional do enfermeiro gestor, 0 seu papel facilitador no enfrentamento das dificuldades que emergem no dia a dia, transmitindo segurança e motivação, e minimizando os riscos psicossociais ${ }^{5}$.

Ao longo do ano de 2020, particularmente no contexto hospitalar, tem sido notório um aumento da mortalidade, sendo uma das principais causas a infeção por COVID-196. Tal situação é geradora de aumento de ansiedade nos profissionais de saúde, que associado à prestação de cuidados perante uma doença transmissível e sem tratamento específico ${ }^{7}$, expõe-nos a maior contato com situações de morte de clientes $^{8}$. De fato, as condições associadas à rápida transmissibilidade do vírus e a necessidade da diminuição dos contatos com os clientes internados, desencadearam mudanças nas dinâmicas de interação e relação, com especial destaque para a separação do cliente e da família. Perante a impossibilidade de existirem visitas, a ausência de despedidas no momento da morte, foi uma realidade vivenciada por muitos profissionais, que em algumas situações se envolveram emocionalmente com a angústia dos familiares e dos clientes ${ }^{7,8}$.

Perante este cenário, além dos desafios, acrescem outras circunstâncias que exigem dos enfermeiros uma nova 
abordagem, até mesmo no que se refere ao direito do cliente a escolher o local e as pessoas que deseja que 0 acompanhem na fase final da vida?.

A par da influência das várias alterações relacionadas com a resposta à pandemia, as atitudes dos enfermeiros frente à morte também influenciam a forma como prestam cuidados aos doentes na fase final da vida. Ao exercer a função de enfermeiro gestor, as atitudes face à morte são ainda mais relevantes, na medida em que tem um papel determinante na orientação da equipe que lidera ${ }^{4}$.

Face às inúmeras exigências, muitas unidades de cuidados foram transformadas para receber doentes com SARS-CoV-2. Perante essa realidade, importa questionar se os enfermeiros gestores estão preparados para orientar a equipe perante as circunstâncias da morte provocada pela COVID-19. Embora a resposta a esta questão seja complexa, é relevante indagar sobre os aspectos que possam ser determinantes na atuação destes profissionais, sendo que um dos primeiros passos, consiste em conhecer as atitudes dos enfermeiros gestores face à morte ${ }^{10}$. Estas podem ser perspectivadas como positivas ou negativas. Quanto às positivas, destacam-se três tipos: aceitação como aproximação, aceitação neutral/neutralidade e aceitação como escape. Em relação às atitudes negativas, prevalecem o medo e o evitamento ${ }^{10}$.

Cada pessoa experiencia a morte e o processo de morrer de uma forma ímpar, e embora seja conhecido que as atitudes dos enfermeiros se refletem na sua atuação, questiona-se no atual contexto pandêmico se as atitudes dos enfermeiros gestores serão facilitadoras da vivência da morte e do processo de morrer.

Perante esta inquietação, integrado em uma investigação mais ampla que se iniciou em 2017, este estudo tem como objetivo analisar as atitudes dos enfermeiros gestores face à morte, antes e após o período crítico da pandemia por COVID-19.

\section{MÉTODO}

Estudo quantitativo, transversal e comparativo, concretizado num Centro Hospitalar do Norte de Portugal. A coleta de dados ocorreu em dois momentos distintos: o primeiro momento entre fevereiro e março de 2018 e o segundo momento em maio de 2020, ou seja, após o primeiro período crítico da pandemia por COVID-19 em Portugal. 
Considerou-se como período crítico aquele em que houve um maior número de doentes internados por COVID-19. Definiram-se como critérios de inclusão no estudo: ser enfermeiro gestor $\mathrm{e}$ exercer a atividade profissional nos serviços de internamento de adultos dos departamentos de medicina, cirurgia e medicina intensiva. Foram excluídos todos aqueles que se encontravam ausentes por motivo de licença, no momento da coleta de dados. De uma população de 54 enfermeiros gestores, no primeiro momento, a amostra ficou constituída por 28 participantes e, no segundo momento, por 21 , dos quais 11 , exerceram funções em serviços de atendimento a doentes com COVID-19.

Importa referir que os 21 enfermeiros gestores, que participaram no segundo momento, já tinham integrado o estudo em 2018, encontrando-se atualmente a exercer funções de gestor no mesmo departamento. Os restantes sete enfermeiros gestores, que compuseram a amostra no primeiro momento, em 2020 optaram por não participar.

Como instrumento de coleta de dados utilizou-se um questionário de autopreenchimento composto por duas partes: uma referente à caracterização sociodemográfica e profissional dos participantes e, outra, pela Escala de Avaliação do Perfil de Atitudes acerca da Morte (EAPAM). A EAPAM, validada para o contexto português em 201011, é constituída por 32 itens, distribuídos por cinco dimensões: aceitação como aproximação; aceitação como escape; aceitação neutral/neutralidade; medo e evitamento.

A dimensão aceitação como aproximação inclui 10 itens: 4 - Acredito que depois de morrer irei para o céu; 8 A morte é a entrada num lugar último de satisfação; 13 - Acredito que o céu será um lugar muito melhor do que este mundo; 15 - A morte é a união com Deus e a felicidade eterna; 16 - A morte traz a promessa de uma vida nova e gloriosa; 22 - Olho para o futuro, depois da morte, como a reunião com as pessoas que amei; 25 - Vejo a morte como uma passagem para um lugar eterno e santo; 27 - A morte oferece uma maravilhosa libertação da alma; 28 - Uma coisa que me dá conforto face à morte são as minhas crenças; 31 - Olho antecipadamente para a vida depois da morte.

A dimensão aceitação como escape é composta por cinco itens: 5 - A morte traz um fim para todos os meus problemas; 9 - A morte providencia um escape para este mundo terrível; 11 - A 
morte é a libertação da dor e do sofrimento; 23 - Vejo a morte como um alívio para o sofrimento terreno; 29 Vejo a morte como alívio do fardo desta vida.

A dimensão aceitação neutral/neutralidade inclui cinco itens: 6 - A morte deve ser vista como um acontecimento natural, inegável e inevitável; 14 - A morte é um aspecto natural da vida; 17 - Eu não temo a morte nem a desejo; 24 - A morte é simplesmente uma parte do processo da vida; 30 - A morte não é boa nem má.

A dimensão medo é constituída por sete itens: 1 - A morte é sem dúvida uma experiência cruel; 2 - A perspectiva da minha própria morte provoca-me ansiedade; 7 - Incomodo-me com a finalidade da morte; 18 - Tenho um intenso medo da morte; 20 - 0 assunto da vida depois da morte perturba-me muito; 21 - Assusta-me o fato da morte significar o fim de tudo o que eu conheço; 32 - A incerteza de nada saber ao que acontece depois da morte.

Por fim, a dimensão evitamento inclui cinco itens: 3 - Evito a todo custo pensamentos relacionados com a morte; 10 - Sempre que um pensamento relacionado com a morte me vem à cabeça tento afastá-lo a todo custo; 12 Tento sempre não pensar na morte; 19 -
Evito a todo custo pensar acerca da morte; 26 - Tento não fazer nada que esteja relacionado com o assunto da morte.

Importa referir que a resposta a cada item está pontuada em escala tipo Likert em que 1 corresponde a "discordo completamente", 2 “discordo", 3 "discordo moderadamente", 4 "não concordo, nem discordo", 5 "concordo moderadamente", 6 "concordo" e 7 "concordo completamente". 0 score total da EAPAM pode variar entre $32 \mathrm{e}$ 224. Na amostra em estudo, a EAPAM obteve um alfa Cronbach de 0,880.

0 estudo obteve a aprovação da comissão de ética do Centro Hospitalar com o número 102/2017 e a adenda de extensão ao trabalho teve aprovação a 26 de maio de 2020. A coleta de dados foi feita por um dos investigadores. Em cada departamento em estudo, foram entregues os questionários correspondentes ao número de enfermeiros gestores e, posteriormente, recolhidos no local, mediante agendamento prévio e disponibilidade dos profissionais.

Os enfermeiros gestores foram também esclarecidos sobre os objetivos e informados de que a sua participação seria voluntária, não sendo prejudicados se não quisessem participar ou em caso 
de desistência. Todos os que aceitaram participar assinaram o termo de consentimento informado. Foi garantida a confidencialidade e o anonimato na utilização e divulgação dos dados obtidos.

Para a análise dos dados, recorreu-se à estatística descritiva e inferencial, com auxílio do programa Statistical Package for the Social Sciences(SPSS), versão 22.0. Para calcular o grau de concordância ou discordância dos enfermeiros gestores com os diferentes itens que compõem a escala, optou-se por considerar como concordância o somatório dos itens 5 (concordo moderadamente), 6 (concordo) e 7 (concordo completamente), e como discordância o somatório dos itens 1 (discordo completamente), 2 (discordo) e 3 (discordo moderadamente). De modo a verificar se existiam diferenças estatisticamente significativas $(p<0,05)$ entre as cinco dimensões da EAPAM, antes e após o período crítico da pandemia por COVID-19 em Portugal, recorreu-se ao teste $T$ Student.

\section{RESULTADOS}

Em relação à caracterização sociodemográfica e profissional, os 28 enfermeiros gestores que participaram no primeiro momento de coleta de dados eram predominantemente do sexo feminino $(71,4 \% ; n=20)$ e casados ou em união de fato $(64,3 \% ; n=18)$. Quanto à área de trabalho, 39,3\% ( $n=11)$ exerciam funções em serviços do departamento de medicina intensiva, $35,7 \% \quad(n=10)$ em serviços do departamento de medicina e $25,0 \% \quad(n=7) \quad$ em serviços do departamento de cirurgia.

Relativamente ao segundo momento de coleta de dados, os 21 enfermeiros gestores também eram maioritariamente do sexo feminino $(61,9 \% ; n=13)$ e casados ou em união de fato $(65,0 \% ; n=13)$. Importa referir que $52,4 \%(n=11)$ dos enfermeiros gestores que participaram no segundo momento de coleta de dados, trabalhavam em áreas de atendimento a doentes com COVID-19, 36,4\% ( $n=4)$ em serviços do departamento de medicina intensiva, e igual percentagem nos serviços do departamento de medicina $(27,3 \%=3)$ e nos serviços do departamento de cirurgia $(27,3 \%=3)$ e $9,1 \%(n=1)$ noutro local.

Pela análise à concordância e discordância manifestada pelos participantes em relação às atitudes medo e evitamento da EAPAM (Tabela 1), verificou-se que no que se refere ao medo, no ano de 2020 os itens "a morte 
é sem dúvida uma experiência cruel” (Item 1) e "a perspectiva da minha própria morte provoca-me ansiedade" (Item 2), foram os que obtiveram maior concordância dos participantes. Este último foi também o que obteve maior concordância em 2018.

No que respeita à maior discordância, no ano de 2020 ela situouse no item "o assunto da vida depois da morte perturba-me muito" (Item 20). Em 2018, houve alguma mudança de perspectiva, pois, nessa data, a maior discordância remetia para o item "tenho um intenso medo da morte" (Item 18).

Em relação ao evitamento, no ano de 2020, verificou-se que os enunciados "evito a todo custo pensamentos relacionados com a morte" (Item 3) e "evito a todo custo pensar acerca da morte" (Item 19), foram aqueles em que se obteve maior concordância dos participantes. Em 2018, a concordância mais elevada foi no item "tento sempre não pensar na morte" (Item 12).

0 item 26, "tento não fazer nada que esteja relacionado com o assunto da morte", foi o que obteve maior discordância, tanto em 2020, como em 2018.

Relativamente à concordância e discordância manifestada pelos participantes em relação às atitudes Aceitação como Aproximação, Aceitação como Escape e Aceitação Neutral/Neutralidade (Tabela 2), apurou-se que, no que concerne à atitude Aceitação como Aproximação, o item 28 "uma coisa que me dá conforto face à morte são as minhas crenças" foi o que obteve maior concordância em 2020 e em 2018. Os itens que registraram maior discordância em 2020 foram "acredito que o céu será um lugar muito melhor do que este mundo" (Item 13) e "olho antecipadamente para a vida depois da morte" (Item 31). Em 2018, o item 31 foi também o que apresentou maior discordância.

No que concerne à atitude Aceitação como Escape, o item 11 "a morte é a libertação da dor e do sofrimento" foi a que registrou maior concordância em 2020 e em 2018. Além do item anterior, em 2018, a concordância foi também elevada no item 23 "vejo a morte como um alívio para o sofrimento terreno". Relativamente à discordância, em 2020, os itens 9 "a morte providencia um escape para este mundo terrível" e 29 "vejo a morte como alívio do fardo desta vida", foram os que obtiveram um valor mais elevado. 0 item 9 foi também o que obteve maior discordância em 2018. 
Tabela 1 - Concordância e discordância relativa às atitudes de medo e evitamento acerca da morte, antes e depois do período crítico da COVID-19. Porto, Portugal, 2020.

\begin{tabular}{|c|c|c|c|c|c|c|c|c|}
\hline$\stackrel{\mathscr{c}}{\Phi}$ & $\begin{array}{c}\text { Coleta de } \\
\text { dados }\end{array}$ & $\begin{array}{c}1^{*} \\
n(\%)\end{array}$ & $\begin{array}{c}2 * \\
n(\%)\end{array}$ & $\begin{array}{c}3 * \\
n(\%)\end{array}$ & $\begin{array}{c}4^{*} \\
n(\%)\end{array}$ & $\begin{array}{c}5^{*} \\
n(\%)\end{array}$ & $\begin{array}{c}\text { 6* } \\
n(\%)\end{array}$ & $\begin{array}{c}7^{*} \\
n(\%)\end{array}$ \\
\hline \multicolumn{9}{|c|}{ Medo } \\
\hline \multirow{2}{*}{1} & 2018 & $1(3,6)$ & $6(21,4)$ & $1(3,6)$ & $3(10,7)$ & $3(10,7)$ & $7(25,0)$ & $7(25,0)$ \\
\hline & 2020 & $0(0)$ & $4(19,0)$ & $0(0,0)$ & $1(4,8)$ & $3(14,3)$ & $9(42,9)$ & $4(19,0)$ \\
\hline \multirow{2}{*}{2} & 2018 & $1(3,6)$ & $2(7,1)$ & $3(10,7)$ & $1(3,6)$ & $7(25,0)$ & $12(42,9)$ & $2(7,1)$ \\
\hline & 2020 & $1(4,8)$ & $2(9,5)$ & $1(4,8)$ & $1(4,8)$ & $6(28,6)$ & $7(33,3)$ & $3(14,3)$ \\
\hline \multirow{2}{*}{7} & 2018 & $1(3,6)$ & $5(17,9)$ & $2(7,1)$ & $11(39,3)$ & $3(10,7)$ & $5(17,9)$ & $1(3,6)$ \\
\hline & 2020 & $3(14,3)$ & $3(14,3)$ & $2(9,5)$ & $5(23,8)$ & $5(23,8)$ & $3(14,3)$ & $0(0)$ \\
\hline \multirow{2}{*}{18} & 2018 & $2(7,1)$ & $13(46,4)$ & $5(17,9)$ & $3(10,7)$ & $2(7,1)$ & $3(10,7)$ & $0(0)$ \\
\hline & 2020 & $2(9,5)$ & $5(23,8)$ & $1(4,8)$ & $3(14,3)$ & $6(28,6)$ & $2(9,5)$ & $2(9,5)$ \\
\hline \multirow{2}{*}{20} & 2018 & $7(25,0)$ & $7(25,0)$ & $1(3,6)$ & $8(28,6)$ & $3(10,7)$ & $1(3,6)$ & $1(3,6)$ \\
\hline & 2020 & $4(19,0)$ & $4(19,0)$ & $1(4,8)$ & $7(33,3)$ & $2(9,5)$ & $3(14,3)$ & $0(0)$ \\
\hline \multirow{2}{*}{21} & 2018 & $1(3,6)$ & $6(21,4)$ & $4(14,3)$ & $0(0)$ & $6(21,4)$ & $10(35,7)$ & $1(3,6)$ \\
\hline & 2020 & $1(4,8)$ & $4(19,0)$ & $2(9,5)$ & $4(19,0)$ & $4(19,0)$ & $4(19,0)$ & $2(9,5)$ \\
\hline \multirow{2}{*}{32} & 2018 & $2(7,1)$ & $7(25,0)$ & $3(10,7)$ & $9(32,1)$ & $2(7,1)$ & $4(14,3)$ & $1(3,6)$ \\
\hline & 2020 & $3(14,3)$ & $2(9,5)$ & $2(9,5)$ & $7(33,3)$ & $3(14,3)$ & $4(19,0)$ & $0(0)$ \\
\hline \multicolumn{9}{|c|}{ Evitamento } \\
\hline \multirow{2}{*}{3} & 2018 & $3(10,7)$ & $11(39,3)$ & $4(14,3)$ & $3(10,7)$ & $3(10,7)$ & $3(10,7)$ & $1(3,6)$ \\
\hline & 2020 & $2(9,5)$ & $5(23,8)$ & $1(4,8)$ & $3(14,3)$ & $4(19,0)$ & $5(23,8)$ & $1(4,8)$ \\
\hline \multirow{2}{*}{10} & 2018 & $2(7,1)$ & $11(39,3)$ & $4(14,3)$ & $3(10,7)$ & $3(10,7)$ & $5(17,9)$ & $0(0)$ \\
\hline & 2020 & $5(23,8)$ & $4(19,0)$ & $2(9,5)$ & $1(4,8)$ & $1(4,8)$ & $7(33,3)$ & $1(4,8)$ \\
\hline \multirow{2}{*}{12} & 2018 & $1(3,6)$ & $12(42,9)$ & $1(3,6)$ & $4(14,3)$ & $5(17,9)$ & $4(14,3)$ & $1(3,6)$ \\
\hline & 2020 & $2(9,5)$ & $7(33,3)$ & $2(9,5)$ & $1(4,8)$ & $2(9,5)$ & $5(23,8)$ & $2(9,5)$ \\
\hline \multirow{2}{*}{19} & 2018 & $2(7,1)$ & $11(39,3)$ & $3(10,7)$ & $5(17,9)$ & $5(17,9)$ & $2(7,1)$ & $0(0,0)$ \\
\hline & 2020 & $3(14,3)$ & $5(23,8)$ & $1(4,8)$ & $2(9,5)$ & $6(28,6)$ & $3(14,3)$ & $1(4,8)$ \\
\hline \multirow{2}{*}{26} & 2018 & $7(25,0)$ & $12(42,9)$ & $4(14,3)$ & $3(10,7)$ & $0(0,0)$ & $2(7,1)$ & $0(0,0)$ \\
\hline & 2020 & $5(23,8)$ & $6(28,6)$ & $1(4,8)$ & $4(19,0)$ & $3(14,3)$ & $1(4,8)$ & $1(4,8)$ \\
\hline
\end{tabular}

*Legenda: 1 - Discordo completamente; 2 - Discordo; 3 - Discordo moderadamente; 4 - Não concordo, nem discordo; 5 - Concordo moderadamente; 6 - Concordo; 7 - Concordo completamente

No que se refere à atitude Aceitação Neutral/Neutralidade, o item 14 "a morte é um aspecto natural da vida", foi a que obteve maior concordância em 2020 e em 2018. A maior discordância em 2020 foi no item 17 "eu não temo a morte nem a desejo" e em 2018 foi o item 30 "A morte não é boa nem má”. Em relação a esta dimensão, importa acrescentar que em 2020 e em 2018, nenhum participante discordou com a afirmativa "a morte é um aspecto natural da vida" (Item 14). Além disso, em 2020 também nenhum participante discordou da afirmativa "a morte é simplesmente uma parte do processo da vida" (Item 24). 
Tabela 2 - Concordância e discordância relativa às atitudes de aceitação acerca da morte, antes e depois do período crítico da COVID-19. Porto, Portugal, 2020.

\begin{tabular}{|c|c|c|c|c|c|c|c|c|}
\hline$\stackrel{n}{\frac{n}{ \pm}}$ & $\begin{array}{c}\text { Coleta de } \\
\text { dados }\end{array}$ & $\begin{array}{c}1^{*} \\
n(\%)\end{array}$ & $\begin{array}{c}2^{*} \\
n(\%)\end{array}$ & $\begin{array}{c}3^{*} \\
n(\%)\end{array}$ & $\begin{array}{c}4^{*} \\
n(\%)\end{array}$ & $\begin{array}{c}5^{*} \\
n(\%)\end{array}$ & $\begin{array}{c}6^{*} \\
n(\%)\end{array}$ & $\begin{array}{c}7^{*} \\
n(\%)\end{array}$ \\
\hline \multicolumn{9}{|c|}{ Aceitação como Aproximação } \\
\hline \multirow{2}{*}{4} & 2018 & $7(25,0)$ & $1(3,6)$ & $0(0,0)$ & $12(42,9)$ & $6(21,4)$ & $2(7,1)$ & $0(0,0)$ \\
\hline & 2020 & $2(9,5)$ & $3(14,3)$ & $1(4,8)$ & $7(33,3)$ & $3(14,3)$ & $4(19,0)$ & $1(4,8)$ \\
\hline \multirow{2}{*}{8} & 2018 & $2(7,1)$ & $8(28,6)$ & $0(0,0)$ & $12(42,9)$ & $1(3,6)$ & $5(17,9)$ & $0(0,0)$ \\
\hline & 2020 & $3(14,3)$ & $3(14,3)$ & $0(0,0)$ & $11(52,4)$ & $3(14,3)$ & $0(0,0)$ & $1(4,8)$ \\
\hline \multirow{2}{*}{13} & 2018 & $9(32,1)$ & $2(7,1)$ & $0(0,0)$ & $12(42,9)$ & $0(0,0)$ & $5(17,9)$ & $0(0,0)$ \\
\hline & 2020 & $3(14,3)$ & $2(9,5)$ & $2(9,5)$ & $9(42,9)$ & $2(9,5)$ & $2(9,5)$ & $1(4,8)$ \\
\hline \multirow{2}{*}{15} & 2018 & $7(25,0)$ & $4(14,3)$ & $0(0,0)$ & $12(42,9)$ & $1(3,6)$ & $3(10,7)$ & $1(3,6)$ \\
\hline & 2020 & $0(0,0)$ & $1(4,8)$ & $1(4,8)$ & $15(71,4)$ & $2(9,5)$ & $1(4,8)$ & $1(4,8)$ \\
\hline \multirow{2}{*}{16} & 2018 & $11(39,3)$ & $1(3,6)$ & $0(0,0)$ & $13(46,4)$ & $0(0,0)$ & $2(7,1)$ & $1(3,6)$ \\
\hline & 2020 & $2(9,5)$ & $2(9,5)$ & $1(4,8)$ & $12(57,1)$ & $3(14,3)$ & $0(0,0)$ & $1(4,8)$ \\
\hline \multirow{2}{*}{22} & 2018 & $6(21,4)$ & $4(14,3)$ & $0(0,0)$ & $9(32,1)$ & $3(10,7)$ & $5(17,9)$ & $1(3,6)$ \\
\hline & 2020 & $1(4,8)$ & $2(9,5)$ & $1(4,8)$ & $8(38,1)$ & $3(14,3)$ & $5(23,8)$ & $1(4,8)$ \\
\hline \multirow{2}{*}{25} & 2018 & $6(21,4)$ & $4(14,3)$ & $0(0,0)$ & $12(42,9)$ & $1(3,6)$ & $4(14,3)$ & $1(3,6)$ \\
\hline & 2020 & $1(4,8)$ & $3(14,3)$ & $1(4,8)$ & $14(66,7)$ & $0(0,0)$ & $1(4,8)$ & $1(4,8)$ \\
\hline \multirow{2}{*}{27} & 2018 & $3(10,7)$ & $6(21,4)$ & $0(0,0)$ & $14(50,0)$ & $0(0,0)$ & $4(14,3)$ & $1(3,6)$ \\
\hline & 2020 & $1(4,8)$ & $1(4,8)$ & $2(9,5)$ & $12(57,1)$ & $3(14,3)$ & $1(4,8)$ & $1(4,8)$ \\
\hline \multirow{2}{*}{28} & 2018 & $2(7,1)$ & $3(10,7)$ & $2(7,1)$ & $4(14,3)$ & $3(10,7)$ & $10(35,7)$ & $4(14,3)$ \\
\hline & 2020 & $0(0,0)$ & $1(4,8)$ & $2(9,5)$ & $8(38,1)$ & $6(28,6)$ & $2(9,5)$ & $2(9,5)$ \\
\hline \multirow{2}{*}{31} & 2018 & $8(28,6)$ & $6(21,4)$ & $3(10,7)$ & $7(25,0)$ & $2(7,1)$ & $2(7,1)$ & $0(0,0)$ \\
\hline & 2020 & $2(9,5)$ & $4(19,0)$ & $1(4,8)$ & $10(47,6)$ & $2(9,5)$ & $1(4,8)$ & $1(4,8)$ \\
\hline
\end{tabular}

\section{Aceitação como Escape}

\begin{tabular}{|c|c|c|c|c|c|c|c|c|}
\hline \multirow{2}{*}{5} & 2018 & $6(21,4)$ & $7(25,0)$ & $2(7,1)$ & $2(7,1)$ & $3(10,7)$ & $4(14,3)$ & $4(14,3)$ \\
\hline & 2020 & $6(28,6)$ & $3(14,3)$ & $0(0,0)$ & $4(19,9)$ & $1(4,8)$ & $3(14,3)$ & $4(19,0)$ \\
\hline \multirow[b]{2}{*}{9} & 2018 & $6(21,4)$ & $14(50,0)$ & $0(0,0)$ & $3(10,7)$ & $1(3,6)$ & $4(14,3)$ & $0(0,0)$ \\
\hline & 2020 & $7(33,3)$ & $5(23,8)$ & $2(9,5)$ & $3(14,3)$ & $1(4,8)$ & $3(14,3)$ & $0(0,0)$ \\
\hline \multirow{2}{*}{11} & 2018 & $2(7,1)$ & $6(21,4)$ & $2(7,1)$ & $5(17,9)$ & $6(21,4)$ & $5(17,9)$ & $2(7,1)$ \\
\hline & 2020 & $2(9,5)$ & $1(4,8)$ & $4(19,0)$ & $4(19,0)$ & $5(23,8)$ & $4(19,0)$ & $1(4,8)$ \\
\hline \multirow{2}{*}{23} & 2018 & $4(14,3)$ & $6(21,4)$ & $0(0,0)$ & $5(17,9)$ & $7(25,0)$ & $6(21,4)$ & $0(0,0)$ \\
\hline & 2020 & $4(19,0)$ & $6(28,4)$ & $1(4,8)$ & $3(14,3)$ & $3(14,3)$ & $4(19,0)$ & $0(0,0)$ \\
\hline & 2018 & $7(25,0)$ & $7(25,0)$ & $1(3,0)$ & $4(14,3)$ & $4(14,3)$ & $5(17,9)$ & $0(0,0)$ \\
\hline & 2020 & $5(23,8)$ & $6(28,6)$ & $3(14,3)$ & $4(19,0)$ & $0(0,0)$ & $3(14,3)$ & $0(0,0)$ \\
\hline
\end{tabular}

\section{Aceitação Neutral/Neutralidade}

\begin{tabular}{ccccccccc}
6 & 2018 & $0(0,0)$ & $0(0,0)$ & $1(3,6)$ & $0(0,0)$ & $1(3,6)$ & $11(39,3)$ & $15(53,6)$ \\
& 2020 & $1(4,8)$ & $0(0,0)$ & $0(0,0)$ & $1(4,8)$ & $1(4,8)$ & $6(28,6)$ & $12(57,1)$ \\
14 & 2018 & $0(0,0)$ & $0(0,0)$ & $0(0,0)$ & $0(0,0)$ & $1(3,6)$ & $9(32,1)$ & $18(64,3)$ \\
& 2020 & $0(0,0)$ & $0(0,0)$ & $0(0,0)$ & $1(4,8)$ & $1(4,8)$ & $7(33,3)$ & $12(57,1)$ \\
\multirow{3}{*}{17} & 2018 & $0(0,0)$ & $4(14,3)$ & $1(3,6)$ & $5(17,9)$ & $4(14,3)$ & $10(35,7)$ & $4(14,3)$ \\
& 2020 & $1(4,8)$ & $0(0,0)$ & $1(4,8)$ & $4(19,0)$ & $5(23,8)$ & $4(19,0)$ & $6(28,6)$ \\
\multirow{2}{*}{24} & 2018 & $0(0,0)$ & $1(3,6)$ & $0(0,0)$ & $0(0,0)$ & $1(3,6)$ & $9(32,1)$ & $17(60,7)$ \\
& 2020 & $0(0,0)$ & $0(0,0)$ & $0(0,0)$ & $2(9,5)$ & $0(0,0)$ & $7(33,3)$ & $12(57,1)$ \\
30 & 2018 & $1(3,6)$ & $3(10,7)$ & $2(7,1)$ & $6(21,4)$ & $4(14,3)$ & $5(17,9)$ & $7(25,0)$ \\
& 2020 & $0(0,0)$ & $0(0,0)$ & $1(4,8)$ & $5(23,8)$ & $1(4,8)$ & $8(38,1)$ & $6(28,6)$ \\
\hline
\end{tabular}

*Legenda: 1 - Discordo completamente; 2 - Discordo; 3 - Discordo moderadamente; 4 - Não concordo, nem discordo; 5 - Concordo moderadamente; 6 - Concordo; 7 - Concordo completamente

Com recurso ao teste $T$ Student constatou-se que existiam diferenças estatisticamente significativas na atitude
Aceitação como Aproximação (Tabela 3).

Nas dimensões Medo, Evitamento e Aceitação Neutral/Neutralidade, não se 
identificaram

diferenças

estatisticamente significativas, mas a média obtida no momento após o período crítico da pandemia por COVID-

19 foi superior. Na dimensão Aceitação como Escape a média obtida em 2020 foi inferior à de 2018. A média global da escala obtida em 2020 foi superior à de 2018.

Tabela 3 - Resultados relativos às dimensões da EAPAM, nos dois momentos da coleta de dados. Porto, Portugal, 2020.

\begin{tabular}{lcccccc}
\hline EAPAM/Dimensões & $\begin{array}{c}\text { Momento } \\
\text { coleta de } \\
\text { dados }\end{array}$ & $\mathbf{n}$ & Média & $\begin{array}{c}\text { Erro } \\
\text { Desvio }\end{array}$ & $\begin{array}{c}\text { Erro } \\
\text { padrão da } \\
\text { média }\end{array}$ & $\begin{array}{c}\text { Teste de } \\
\text { comparação } \\
\text { de médias }\end{array}$ \\
\hline Valor total da Escala & 2018 & 28 & 125,14 & 27,032 & 5,109 & $0,371^{*}$ \\
Medo & 2020 & 21 & 134,05 & 20,289 & 4,427 & \\
Evitamento & 2018 & 28 & 27,75 & 8,249 & 1,559 & $0,710^{*}$ \\
& 2020 & 21 & 29,29 & 8,504 & 1,856 & \\
Aceitação como & 2018 & 28 & 15,68 & 7,061 & 1,334 & $0,087^{*}$ \\
Aproximação & 2020 & 21 & 18,29 & 8,457 & 1,845 & \\
Aceitação como & 2018 & 28 & 35,07 & 14,767 & 2,791 & $0,036^{*}$ \\
Escape & 2020 & 21 & 39,67 & 10,730 & 2,341 & \\
Aceitação Neutral/ & 2018 & 28 & 17,39 & 8,135 & 1,537 & $0,727^{*}$ \\
Neutralidade & 2020 & 21 & 16,90 & 7,880 & 1,720 & \\
\hline
\end{tabular}

"Teste T Student para igualdade de variâncias, sendo considerado $p<0,05$.

\section{DISCUSSÃO}

Os enfermeiros, no desempenho das suas atividades, são profissionais que lidam diariamente com a morte dos clientes, percebendo a finitude da vida e a inexorabilidade da morte, com representações acerca da sua própria morte $^{12,13}$. Tal fato, tem dificultado a reflexão e discussão sobre a temática da morte, conduzindo a fragilidades na aceitação desta realidade, cada vez mais frequente no contexto hospitalar ${ }^{12}$.

A falta de suporte aos enfermeiros, a carência de apoio das instituições e a escassez de estratégias de enfrentamento, proporcionam a sensação de desamparo e despreparo para lidar diretamente com a morte e o processo de morrer ${ }^{14,15}$. Neste contexto, a figura do enfermeiro gestor assume um especial destaque, na medida em que é responsável pela garantia das melhores práticas profissionais, pela promoção da formação e do desenvolvimento dos profissionais de enfermagem, bem como pelo apoio diário aos mesmos no enfrentamento das dificuldades ${ }^{16}$, de que é exemplo a morte e o processo de morrer. Assim, perante o papel do enfermeiro gestor e a convicção de que as atitudes face à morte influenciam 
aspectos práticos e diários da atuação profissional ${ }^{10}$, tornou-se relevante conhecê-las.

Acresce ainda que as atitudes do enfermeiro gestor frente à morte são determinantes para o acompanhamento dos enfermeiros da equipe que lidera, para a definição do modelo assistencial, bem como para a implementação das estratégias que melhor suportem a forma como os enfermeiros enfrentarão a morte e o processo de morrer ${ }^{15}$. De fato, analisar as atitudes frente à morte pode constituir uma excelente estratégia para refletir sobre a assistência prestada às pessoas nos momentos finais da vida ${ }^{12}$, bem como sobre o planejamento $\mathrm{e}$ implementação de estratégias que minimizem as emoções negativas vivenciadas pelos enfermeiros, independentemente da condição em que exerçam a profissão.

Ao analisar as atitudes dos enfermeiros gestores, antes e após o primeiro período crítico da pandemia por COVID-19 em Portugal, constatou-se que predominou a concordância com a Aceitação Neutral/Neutralidade e o Medo, o que além de evidenciar o entendimento da morte como algo natural, que faz parte da vida ${ }^{10,17}$, reforça, mais uma vez, os sentimentos negativos experienciados perante a morte e o processo de morrer ${ }^{12}$.

Embora em contexto pandêmico, a morte constituísse, em muitas situações, um evento inesperado, a afirmativa de que "a morte é um aspecto natural da vida", foi a que obteve maior concordância nos dois momentos de coleta de dados, sendo simultaneamente aquela em que não se registrou qualquer discordância por parte dos participantes. Em relação ao medo, o item "a perspectiva da minha própria morte provoca-me ansiedade", obteve maior concordância em ambos os momentos, comprovando a ansiedade desencadeada pela própria morte, que se constitui como uma das experiências mais difíceis da vida, com repercussões a nível de saúde mental ${ }^{14,18}$, podendo ser agravadas pela interpretação da morte como um fracasso pessoal. Além das repercussões ao nível pessoal, não podem ser descuradas as consequências de âmbito profissional. A este nível, em estudos anteriores, comprovou-se que o medo interfere na qualidade dos cuidados prestados à pessoa em fim de vida $^{19}$.

Numa significativa parte das culturas, o ser humano tende a considerar a morte como uma experiência cruel. Em tempos de 
pandemia por Covid-19 o medo da morte intensificou-se perante 0 risco de contaminação, e está presente na sociedade global, nomeadamente, no discurso expresso pelos meios de comunicação social e decisores políticos. Nesta conjuntura, compreende-se que comparativamente a 2018 se constate maior concordância entre os enfermeiros gestores com o item "a morte é sem dúvida uma experiência cruel”. Estando na linha da frente do combate à pandemia, a vivenciar de perto a gravidade da doença, o sofrimento e o eminente risco de vida dos doentes, é provável que a vivência da morte seja ainda mais marcante e traumática para estes profissionais. Acresce que os enfermeiros gestores além de terem que lidar com os seus sentimentos, têm de manter o apoio aos enfermeiros da equipe que lidera, que sendo responsáveis pela prestação de cuidados a esses doentes, vivenciam ainda com mais proximidade a morte e o processo de morrer.

Tratando-se de uma profissão em que o enfoque é cuidar do outro, perante as circunstâncias da pandemia, é compreensível que o medo da própria morte ganhe uma expressão diferente daquela que se verificava em 2018.
$\mathrm{Na}$ atitude Aceitação como Aproximação, que compreende a morte como uma passagem, associada a crenças religiosas ${ }^{10}$, nos dois momentos de coleta de dados, a resposta dos participantes foi consensual, tendo maioritariamente assumido que as suas crenças the dão conforto face à morte. Nesta dimensão, as diferenças encontradas entre o primeiro e segundo momento foram significativas, sendo a média obtida após o período crítico da pandemia por COVID-19 superior. Embora não se tenha encontrado evidência que comprove que as crenças e as convicções religiosas facilitam o enfrentamento da morte e do processo de morrer, em contexto pandêmico, considera-se que a sensação de impotência perante 0 agravamento clínico de várias doentes com COVID-19, faz com que os próprios profissionais, na tentativa de experienciarem menos emoções negativas, apoiem-se nas suas crenças e convicções, que se revelam importantes perante a necessidade de diariamente lidar com o incerto ${ }^{20}$.

No que se refere à atitude Aceitação como Escape, a ideia de que a morte representa o fim da dor e do sofrimento, evidenciou menor concordância em 2020 do que em 2018. $\mathrm{Na}$ sequência da pandemia, os 
profissionais de saúde depararam-se com o agravamento súbito e muitas vezes irreversível dos doentes com COVID-1920, - que tem culminado num número crescente de mortes. 0 fato de muitas dessas mortes não terem sido desencadeadas por doenças crônicas, caracterizadas por um agravamento dos sinais e sintomas durante um longo período de tempo, mas sim por exacerbações repentinas relacionadas com a infeção por SARS-CoV-2, poderá justificar os menores scores na atitude aceitação como escape. Efetivamente, em muitos casos, o histórico de dor e sofrimento era recente, dificultando o entendimento da morte como uma forma de cessar os mesmos.

Em relação à atitude Evitamento, a média foi maior em 2020, revelando que não pensar e evitar o diálogo sobre a morte pode ter sido uma estratégia adotada pelos participantes para diminuir o stress, já naturalmente agravado em contexto pandêmico. A maior concordância dos enfermeiros gestores nos itens relativos a evitar pensamentos sobre a morte, vem comprovar o mencionado.

0 item "tento não fazer nada que esteja relacionado com o assunto da morte", foi o que obteve menor concordância entre os enfermeiros gestores em ambos os momentos, até porque decorrente do seu exercício profissional, não é legítimo não fazer nada que esteja relacionado com a morte. Lidar com a morte dos pacientes é um dos maiores desafios enfrentados pelos profissionais de enfermagem, estando frequentemente associado a sentimentos de frustração, perda, tristeza e impotência ${ }^{21}$. Vários estudos comprovam que o fato de o processo de formação dos enfermeiros não contemplar de forma aprofundada as temáticas da morte e do processo de morrer, justifica a existência de lacunas de conhecimento, bem como atitudes de evitamento face às situações de morte com que se deparam no seu quotidiano $^{19,21,22}$.

Em contexto pandêmico, com o aumento do número de mortes, a falta de preparação dos enfermeiros para enfrentar a morte e o processo de morrer ficou ainda mais evidente e apesar das atitudes dos enfermeiros gestores nem sempre se coadunarem com o entendimento da morte como algo natural, é crucial que os mesmos planejem e implementem estratégias de apoio aos enfermeiros da equipe, no sentido de manter a qualidade dos cuidados prestados e promover o bemestar desses profissionais. 
Mesmo assumindo como limitação o fato de o estudo ter sido concretizado apenas em uma instituição hospitalar, acredita-se que a identificação das atitudes do enfermeiro gestor frente à morte, permitirá perspectivar as mudanças necessárias, de modo a que este profissional, assuma um papel de destaque, contribuindo de forma significativa para o preparo e acompanhamento dos enfermeiros da equipe a vivenciar diariamente a morte e o processo de morrer dos clientes.

O acompanhamento diário dos enfermeiros, exige dos gestores uma atenção diferenciada, direcionada para ajudar esses profissionais na gestão emocional perante a morte, bem como na prestação de cuidados adequados à pessoa em fase final da vida ${ }^{12,19}$.

Neste sentido, e ainda que não tenham sido predominantes, as atitudes negativas face à morte apresentadas pelos enfermeiros gestores, vêm, mais uma vez, reforçar a importância de também estes profissionais participarem em iniciativas que garantam a sua capacitação para enfrentar a morte e o processo de morrer ${ }^{12}$. Em estudo recente, um programa de formação sobre gestão emocional se revelou efetivo, na medida em que as atitudes de medo e de neutralidade perante a morte foram as dimensões em que os enfermeiros apresentaram mudanças positivas. No caso do medo da morte, este diminuiu de forma significativa ${ }^{19}$.

\section{CONCLUSÃO}

A morte é cada vez mais frequente no contexto hospitalar e as atitudes face à mesma podem influenciar a atuação dos profissionais, bem como os cuidados que prestam, relevando para a importância de se identificarem essas atitudes, no sentido de definir estratégias de mudança adequadas.

Neste estudo, com relação às atitudes dos enfermeiros gestores frente à morte, predominou a concordância com a "aceitação neutral/neutralidade e o "medo". No que se refere à "aceitação como aproximação", a média obtida foi superior no momento após o período crítico da pandemia por COVID-19, evidenciando a importância das crenças como estratégia facilitadora para o enfrentamento da morte e do processo de morrer.

A convicção de que a morte faz parte do processo natural da vida, potenciará a atuação dos enfermeiros gestores no sentido de capacitarem os enfermeiros da equipe que lideram, a 
prestarem melhores cuidados às pessoas na fase final da vida, mas também a adotarem estratégias que minimizem $o$ seu sofrimento no lidar com a morte e o processo de morrer no seu contexto de trabalho.

Neste sentido, o investimento nesta temática nos cursos de graduação em enfermagem, bem como nos cursos de pós-graduação, nomeadamente, naqueles sobre gestão em enfermagem, garantirá a necessária mudança de paradigma na vivência da morte e do processo de morrer, o que nos contextos da prática hospitalar deverá ser impulsionado pelo enfermeiro gestor.

\section{REFERÊNCIAS}

1. Feiten AM, Coelho TR. Quality management in service organizations: barriers and sucess factors. Rev Adm FACES J. 2019; 18(3):56-71.

2. Ferrer R. Pandemia por Covid-19: el mayor reto de la historia del intensivismo. Med Intensiva. 2020; 44(6):323-324.

3. Direção-Geral da Saúde. Plano Nacional de Preparação e Resposta à Doença por novo coronavírus (COVID19) [Internet]. Lisboa: Direção-Geral da Saúde; 2020 [cited 2020 Jun 01]. Available from: https: //covid19.minsaude.pt/wpcont ent/uploads/2020/03/Plano-de-

Conting\%C3\%AAncia-NovoCoronavirus_Covid-19.pdf

4. Ventura-Silva JMA, Ribeiro OMPL, Santos MR, Faria ACA, Monteiro MAJ, Vandresen L. Planejamento organizacional no contexto de pandemia por COVID-19: implicações para a gestão em enfermagem. J Health NPEPS. 2020; 5(1):e4626.

5. Almeida JA. Internal Medicine in Centro Hospitalar Universitário S. João and the COVID-19 Pandemic. Med Intensiv. 2020; 1-6.

6. Nogueira PJ, Nobre MA, Nicola PJ, Furtado C, Carneiro AV. Excess Mortality Estimation During the COVID-19 Pandemic: Preliminary Data from Portugal. Acta Med Port. 2020; 33(6):376-383.

7. Soares SSS, Almeida ASS, Jesus LS, Carvalho EC, Varella TC, Souza NVDO. Covid-19 pandemic and the death/dying process: reflections on nursing students. RSD. 2020; 9(9):e615997766.

8. Tavares CQ. Dimensões do cuidado na perspectiva da espiritualidade durante a pandemia pelo novo coronavírus (COVID-19). J Health NPEPS. 2020; 5(1):1-4. 
9. Ordem dos Enfermeiros. Código Deontológico dos Enfermeiros [Internet]. Lisboa: Ordem dos Enfermeiros; 2012 [cited 2020 Sept 30]. Available from: https: / /www.ordemenfermeiros.pt/ media/8889/codigodeontologicoenfe rmeiro_edicao2005.pdf

10. Machado RS, Oriá MOB, Fernandes MA, Gouveia MTO, Silva GRF. Translation and cultural adaptation of death attitude profile Revised (DAP-R) for use in Brazil. Texto \& contexto enferm.

2019; 28:e20180238.

11. Loureiro LMJ. Translation and adaptation of the Revised Death Attitude Profile (DAP-R). Rev Enf Ref. 2010; III(1):101-108.

12. Cardoso MFPT, Martins MMFPS, Ribeiro OMPL, Fonseca EF. Nurses' attitudes towards death in the hospital context: differentiation by care units. Esc Anna Nery Rev Enferm. 2021; 25(1):e20200100.

13. Barbosa AGC, Massaroni L, Lima EFA. Significados do processo do morrer e da morte para a equipe multiprofissional. Rev Pesqui (Univ Fed Estado Rio J Online). 2016; $8(2): 4510-7$.

14. Povedano-Jimenez M, GranadosGamez G, Garcia-Caro MP. Work environment factors in coping with patient death among Spanish nurses: a cross-sectional survey. Rev latinoam enferm. 2020; 28:e3234.

15. Perboni JS, Zilli F, Oliveira SG. Health professionals and the patient death and die process: an integrating review. Pers bioét. 2018; 22(2):288302.

16. Ordem dos Enfermeiros. Regulamento do Perfil de Competências do Enfermeiro Gestor [Internet]. Lisboa: Ordem dos Enfermeiros; 2015. Available from: https://dre.pt/application/conteudo /114599547

17. Souza MCS, Sousa JM, Lago DMSK, Borges MS, Ribeiro LM, Guilhem DB. Evaluation of the Death Attitude Profile-Revised: a study with health science undergraduate students. Texto \& contexto enferm. 2017; 26(4):e3640016.

18. Tabar M. Investigating the status of life satisfation quality of life and death anxiety according to the personality traits of the old living at home and city of Kermanshah. Int Educ Res J. 2018; 4(4):91-95.

19. Pais NJ, Costeira CR, Silva AM, Moreira IM. Efetividade de um programa de formacao na gestao emocional dos enfermeiros perante a 
morte do doente. Rev Enf Ref. 2020; 5(3):e20023.

20. Silva MCQS, Vilela ABA, Silva RS, Boery RNSO. The dying process and death of patients with COVID-19: a reflection in the light of spirituality. Cogitare enferm. 2020; 25:e73571.

21. Gonçalves MJR, Simões JRS. The importance of the nursing team in preventing diabetic foot. Rev J'RG. 2019; 2(5):166-182.

22. Lopes MFGL, Melo YST, Santos MWC, Oliveira DAL, Maciel AM. Vivências de enfermeiros no cuidado às pessoas em processo de finitude. Rev Ciênc Plur. 2020; 6(2):82-100.

Financiamento: Os autores declaram que não houve financiamento.

Conflito de interesses: Os autores declaram não haver conflito de interesses.

\section{Participação dos autores:}

- Concepção: Cardoso MFPT, Martins MMFPS, Ribeiro OMPL.

- Desenvolvimento: Cardoso MFPT, Martins MMFPS, Ribeiro OMPL, Pereira VLSC, Pires RMF, Santos MR.

- Redação e revisão: Cardoso MFPT, Martins MMFPS, Ribeiro OMPL, Pereira VLSC, Pires RMF, Santos MR.

Como citar este artigo: Cardoso MFPT, Martins MMFPS, Ribeiro OMPL, Pereira VLSC, Pires RMF, Santos MR. Atitude dos enfermeiros gestores face à morte: repercussões da pandemia por COVID-19. J Health NPEPS. 2020; 5(2):42-59.

Submissão (Fast Track COVID-19): 22/10/2020

Aceito (Fast Track COVID-19): 26/10/2020

Publicado (Fast Track COVID-19): 26/10/2020 